found that PDSs could be generated in the absence of action potentials; indeed, 70-90\% of PDSs were insensitive to tetrodotoxin, a sodium-channel blocker. Glutamate is thought to be central to the development of epileptiform activity, so the researchers looked at action potential-independent glutamate release. They photo-released caged astrocytic $\mathrm{Ca}^{2+}$ to observe the effect of increased $\mathrm{Ca}^{2+}$-dependent glutamate release from astrocytes. In most cases, increased astrocytic $\mathrm{Ca}^{2+}$ triggered a PDS.

Artificially evoked astrocytic $\mathrm{Ca}^{2+}$ waves were used to assess the effects of antiepileptic drugs on astrocytic $\mathrm{Ca}^{2+}$ signaling in nonepileptic live mice. In all cases, $\mathrm{Ca}^{2+}$ wave propagation was reduced-valproate decreased signaling by $65 \%$, and gabapentin decreased signaling by $54 \%$.

The authors suggest that an action potentialindependent source of glutamate can generate local depolarization and synchronized firing, and that anticonvulsant drugs might in part act by depressing astrocytic activity. Drugs targeted at suppressing astrocytic $\mathrm{Ca}^{2+}$ signaling might in future be able to control seizures while leaving normal neural transmission undisturbed.

Christine Kyme

Original article Tian G-F et al. (2005) An astrocytic basis of epilepsy. Nat Med 11: 973-981

\section{REACH enables treatment of acute stroke in rural hospitals}

A lack of resources in US rural hospitals often leads to acute stroke patients being treated inadequately or having to travel to distant regional hospitals, thereby delaying treatment. To overcome these problems, a recent study advocates use of the REACH telestroke system, which enables rural doctors and distant stroke specialists to interact over a broadband Internet connection. The specialist can access CT scans and patient video clips to calculate stroke severity and make recommendations for treatment. Advice can be given on the suitability for each patient of the thrombolytic agent tissue plasminogen activator (tPA), early use of which has been shown to correlate with better outcome.

Hess et al. reviewed 194 stroke patients evaluated using the REACH system between March 2003 and May 2005 in a network of eight rural hospitals in Georgia, USA. The time from stroke onset to treatment with IPA was a mean of $122 \mathrm{~min}$; this decreased as users' familiarity with the system increased. Nearly a quarter of the 30 patients given tPA were treated within $\leq 90 \mathrm{~min}$ of admission, and more than half within $2 \mathrm{~h}$. CT or MRI scans were carried out within $36 \mathrm{~h}$ of tPA administration. Patient response was good, with low in-hospital mortality, no symptomatic intracerebral hemorrhages, and marked improvement within $24 \mathrm{~h}$ in $60 \%$ of tPA-treated patients.

By providing access to a 24-hour stroke team, the REACH system enables rural hospitals to provide rapid, safe and effective treatment with tPA. The costs of setting up and maintaining the system are low, making it an extremely feasible option.

Pippa Murdie

Original article Hess DC et al. (2005) REACH: clinical feasibility of a rural telestroke network. Stroke 36: 2018-2020

\section{Extreme premature birth and risk of cerebellar hemorrhage}

A recent retrospective study by Limperopoulos and colleagues examined the incidence and topography of cerebellar hemorrhage $(\mathrm{CBH})$, and its risk factors, in premature infants. Their results indicate that the condition is an under-recognized complication of extreme premature birth.

The researchers searched a database listing infants admitted to a neonatal intensive care unit between 1998 and 2002. Out of a total of 1,242 preterm infants ( $<37$ weeks' gestation), they identified 35 who had been diagnosed with $\mathrm{CBH}$ using neonatal cranial ultrasound and who were not excluded from the study because of conditions such as known or suspected brain malformation. Cases were double-matched with 70 preterm control infants. Clinical data were collected from medical records regarding, prenatal, intrapartum and early postnatal outcomes.

Eight of the 35 infants were found to have isolated $\mathrm{CBH}$, and combined $\mathrm{CBH}$ and supratentorial extraparenchymal or parenchymal hemorrhage was identified in the remaining 27 infants. Univariate analysis revealed that assisted conception was associated with an almost five-fold higher risk of infant $\mathrm{CBH}(P=0.006)$, and multiple regression analysis demonstrated that emergent cesarean section independently increased the odds of $\mathrm{CBH}$ eight-fold, and patent ductus arteriosus almost seven-fold.

The authors conclude that risk factors for $\mathrm{CBH}$ appear to be multifactorial and that the condition is currently underappreciated in 